\title{
Knockdown of IncRNA-HOTAIR downregulates the drug-resistance of breast cancer cells to doxorubicin via the PI3K/AKT/mTOR signaling pathway
}

\author{
ZHIXIANG LI, JUN QIAN, JING LI and CHAO ZHU \\ Department of Tumor Surgery, The First Affiliated Hospital of Bengbu Medical College, Bengbu, Anhui 233000, P.R. China
}

Received August 15, 2018; Accepted February 5, 2019

DOI: $10.3892 /$ etm.2019.7629

\begin{abstract}
The resistance to chemotherapeutic drugs is a critical feature of breast cancer recurrence and metastasis. Long non-coding RNAs (LncRNAs) serve key roles in tumor drug resistance. LncRNA-HOX transcript antisense RNA (HOTAIR) has been reported to be overexpressed in certain types of cancer and may be closely associated with tumor resistance. The current study aimed to investigate the role of lncRNA-HOTAIR in the regulation of breast cancer resistance to doxorubicin (DOX). A breast cancer cell line (MCF-7) and DOX-resistant breast cancer cell line (DOXR-MCF-7) were utilized in the current study. DOXR-MCF-7 cells were transfected with lncRNA-HOTAIR small interfering RNA (siRNA) and control siRNA. Subsequently, MTT and colony formation assays were performed to assess cell proliferation. Cell apoptosis was also evaluated via flow cytometry. In addition, western blotting and reverse transcription-quantitative polymerase chain reaction were performed to detect the expression of caspase-3, B-cell lymphoma 2, Bcl-2-associated $\mathrm{X}$ protein, phosphoinositide 3-kinase (PI3K), protein kinase $\mathrm{B}$ (AKT) and mechanistic target of rapamycin (mTOR), and the phosphorylation of PI3K, AKT, and mTOR. The data indicated that lncRNA-HOTAIR silencing decreased cell proliferation and increased apoptosis in MCF-7 and DOXR MCF-7 cells. Furthermore, lncRNA-HOTAIR silencing significantly decreased the phosphorylation of PI3K, AKT and mTOR, indicating that the knockdown of IncRNA-HOTAIR effectively attenuates the resistance of breast cancer cells to DOX by inhibiting the PI3K/AKT/mTOR pathway. In summary, the present study indicated that the knockdown of IncRNA-HOTAIR weakened the resistance of breast cancer cells to DOX via PI3K/AKT/mTOR signaling, suggesting
\end{abstract}

Correspondence to: Dr Zhixiang Li, Department of Tumor Surgery, The First Affiliated Hospital of Bengbu Medical College, 287 Yan Huai Road, Bengbu, Anhui 233000, P.R. China

E-mail: lizhixiang126@163.com

Key words: breast cancer, doxorubicin resistance, long non-coding RNA, HOX transcript antisense RNA, phosphoinositide 3-kinase that lncRNA-HOTAIR may be a novel intervention target to reverse DOX-resistance in breast cancer.

\section{Introduction}

Breast cancer is a common malignant tumor with an increasing incidence rate $(1,2)$. According to the World Cancer Research Fund, 450,000 mortalities are caused by breast cancer worldwide each year, accounting for $13.7 \%$ of cancer-associated mortalities in women (3). Great efforts have been made to improve the diagnosis and treatment of breast cancer in recent years; however, the pathogenic mechanism of breast cancer is yet to be fully elucidated. At present, doxorubicin (DOX) is considered to be one of the most effective drugs for the treatment of breast cancer (4). However, following several treatments, drug resistance may develop, which leads to therapy failure $(5,6)$. Currently, the mechanism of DOX resistance is poorly understood.

Long non-coding RNA (lncRNA) are RNA molecules comprising $>200$ nucleotides that do not possess any protein-coding capacity $(7,8)$. However, lncRNAs serve key roles in the transcriptional, post-transcriptional and epigenetic regulation of gene expression (9). Numerous studies have indicated that the abnormal expression of lncRNA is associated with various tumor functions, including tumor formation, drug resistance and metastasis $(10,11)$. Additionally, the abnormal expression of the lncRNA, HOX transcript antisense RNA (HOTAIR), was identified in breast cancer cell lines and tissue $(12,13)$. A previous study demonstrated that lncRNA-HOTAIR increased cisplatin resistance in gastric cancer by targeting microRNA-126 via the phosphoinositide 3-kinase (PI3K)/protein kinase B (AKT)/multidrug resistanceassociated protein 1 (MRP1) pathway (14). However, It is not clear whether LncRNA-HOTAIR is involved in resistance to DOX in breast cancer cells.

The PI3K/AKT/mechanistic target of rapamycin (mTOR) signal transduction pathway serves a key role in the cell cycle, the proliferation of cells, cellular metabolism and in protein synthesis (15). A previous study has indicated that the PI3K/AKT/mTOR pathway is frequently activated in ovarian cancer (16). Furthermore, the aberrant activation of the signaling pathway is associated with the development of breast cancer and has therefore been targeted for the treatment of breast cancer (17). 
The present study aimed to assess whether lncRNAHOTAIR is associated with the resistance of breast cancer cells to DOX. The results revealed that the silencing of lncRNA-HOTAIR decreased the resistance of breast cancer cells to DOX by suppressing the PI3K/AKT/mTOR pathway. These results may provide a novel intervention target to reverse DOX-resistance in breast cancer.

\section{Materials and methods}

Cell culture. The human breast cancer cell lines, MCF-7 and SKBR3, were obtained from the American Type Culture Collection (Manassas, VA, USA). Cells were cultivated in RPMI-1640 medium (Gibco; Thermo Fisher Scientific, Inc., Waltham, MA, USA) supplemented with $10 \%$ fetal bovine serum (Gibco; Thermo Fisher Scientific, Inc.) and incubated at $37^{\circ} \mathrm{C}$ with $5 \% \mathrm{CO}_{2}$.

Establishment of DOX-resistant cell lines. DOX was purchased from the First Affiliated Hospital of Kunming Medical University (Kunming, China). MCF-7 and SKBR3 cells were cultured in DOX-free Dulbecco's modified Eagle's medium (DMEM; Gibco; Thermo Fisher Scientific, Inc.) until cells were in the logarithmic growth phase, at which point the culture medium was replaced with DMEM medium containing a low dose of DOX $(0.2 \mu \mathrm{g} / \mathrm{ml})$. Subsequently, the concentration of DOX was increased from 0.2 to $0.6 \mu \mathrm{g} / \mathrm{ml}$ in $0.04 \mu \mathrm{g}$ increments every 3 weeks. The DOX-resistant MCF-7 and SKBR 3 cell lines were considered to have been successfully established when cells were able to survive under high DOX treatment $(0.6 \mu \mathrm{g} / \mathrm{ml})$. Prior to subsequent experimentation, cells were cultured in the drug-free DMEM medium containing $5 \% \mathrm{CO}_{2}$ at $37^{\circ} \mathrm{C}$ for 2 days.

Cell transfection and RNA interference. LncRNA-HOTAIR small interfering RNAs (siRNAs or siR; siR-HOTAIR1, 3'-GAACGGGAGUACAGAGAGAUU-5'; siR-HOTAIR1-2, 3'-CCACAUGAACGCCCAGAGAUU-5') were designed and synthesized by Shanghai GenePharma Co., Ltd. (Shanghai, China). Negative control siRNA (3'-CUACAACAGCCA CAACGUCdTdT-5') was purchased from Sangon Biotech Co., Ltd. (Shanghai, China). Briefly, $500 \mathrm{ng}$ siR-HOTAIR1 was transfected into MCF-7 cells and DOXR-MCF-7 cells using $1 \mu 1$ Lipofectamine ${ }^{\circledR} 2000$ (Invitrogen; Thermo Fisher Scientific, Inc.) according to the manufacturer's protocol. The cells were incubated at $37^{\circ} \mathrm{C}$ in an atmosphere containing 5\% $\mathrm{CO}_{2}$ for $6 \mathrm{~h}$. Subsequently, transfected cells were incubated at $37^{\circ} \mathrm{C}$ for $72 \mathrm{~h}$. Cells were then divided into the following experimental groups: Untransfected MCF-7 cells, negative control siRNA-transfected MCF-7 cells, siR-HOTAIR1 transfected MCF-7 cells; untransfected DOXR-MCF-7 cells, negative control siRNA-transfected DOXR-MCF-7 cells and siR-HOTAIR1 transfected DOXR-MCF-7 cells.

Reverse transcription-quantitative polymerase chain reaction (RT-qPCR) analysis. Total RNA was extracted from MCF-7 and DOXR-MCF-7 cells using TRIzol reagent (Thermo Fisher Scientific, Inc.) according to the manufacturer's protocol. Total RNA was then reverse-transcribed into cDNA using an RT kit (Takara Biotechnology Co., Ltd., Dalian, China) following the manufacturer's protocol. qPCR was performed using the SYBR Premix Ex Taq (Applied Biosystems; Thermo Fisher Scientific, Inc.) with the following thermocycling conditions: Initial denaturation at $95^{\circ} \mathrm{C}$ for $10 \mathrm{~min}$, followed by 40 cycles of denaturation for $30 \mathrm{sec}$ at $95^{\circ} \mathrm{C}$, annealing at $60^{\circ} \mathrm{C}$ for $30 \mathrm{sec}$ and extension at $72^{\circ} \mathrm{C}$ for $30 \mathrm{sec}$. Relative expression levels were calculated using the $2^{-\Delta \Delta \mathrm{Cq}}$ method (18). GAPDH was used as the reference gene. The following primers were used for amplification: GAPDH forward, 5'-ATTGATGGATGCTAFGAGTATT-3' and reverse, 5'-AGTCTTCTGGGTGGCAGTGAT-3'; IncRNA-HOTAIR forward, 5'-CGGAGTGAGTTTATCG CAG-3' and reverse, 5'-GGCGACCGGAGCTCATCTTACC-3'.

MTT assay. Following $72 \mathrm{~h}$ incubation, cell proliferation analysis was performed using an MTT assay kit (Sigma-Aldrich; Merck KGaA, Darmstadt, Germany). MCF-7 and DOXR-MCF-7 cells $\left(5 \times 10^{5}\right.$ cells/well) were seeded into 96-well plates. Subsequently, cell proliferation was detected at different time points (24, 48 and $72 \mathrm{~h}$ ). MTT (5 mg/Ml, $20 \mu \mathrm{l}$ ) was added to cells and incubated for $4 \mathrm{~h}$ at $37^{\circ} \mathrm{C}$. The precipitate was dissolved in $100 \mu \mathrm{l}$ dimethyl sulfoxide and absorbance at $490 \mathrm{~nm}$ was detected using a microplate spectrophotometer.

Colony formation assay. A colony formation assay was performed following transfection. Cells (500 cells/well) were seeded into 6-well plates and incubated for 10 days in $5 \% \mathrm{CO}_{2}$ at $37^{\circ} \mathrm{C}$ to form colonies. Cells were fixed in $95 \%$ methanol for $15 \mathrm{~min}$ at $4^{\circ} \mathrm{C}$ and stained with $1 \%$ crystal violet solution for $30 \mathrm{~min}$ at room temperature. Images were captured under a light microscope (magnification, x100). Colony number was quantified using Alpha-View Software 3.1 (FluorChem Q; ProteinSimple, San Jose, CA, USA).

Flow cytometric assay. Cell apoptosis was assessed using a fluorescein isothiocyanate (FITC) apoptosis detection kit (Oncogene Research Products, La Jolla, CA, USA) in accordance with the manufacturer's protocol. Cells were harvested, washed twice with pre-cooled PBS and stained successively with propidium iodide $(10 \mu \mathrm{l})$ and Annexin-V-FITC $(10 \mu \mathrm{l}$; Nanjing KeyGen Biotech Co., Ltd., Nanjing, China). Following 15 min of incubation at room temperature, a flow cytometer (FACSVantage SE; BD Biosciences, San Jose, CA, USA) was used for sample analysis using the FlowJo software package (version 10.0.7; Tree Star, Inc., Ashland, OR, USA). The number of cells in each classification was presented.

Western blot analysis. Western blotting was performed to assess the expression of proteins of interest. Proteins were isolated using radioimmunoprecipitation assay lysis buffer (Beyotime Institute of Biotechnology, Haimen, China) and protein concentration was measured using a bicinchoninic acid protein assay kit (Beyotime Institute of Biotechnology). Proteins (20 $\mu \mathrm{g} / \mathrm{per}$ lane) were subsequently separated via SDS-PAGE on $10 \%$ gels using polyvinylidene fluoride (PVDF) membranes (EMD Millipore, Billerica, MA, USA) for protein transfer. Membranes were then blocked with 5\% nonfat milk for $2 \mathrm{~h}$ at room temperature and incubated with the following primary antibodies obtained from Cell Signaling Technology, Inc. (Danvers, MA, USA): Anti-Bcl-2-associated X protein (Bax; 1:1,000; cat. no. 2774), anti-B-cell lymphoma 

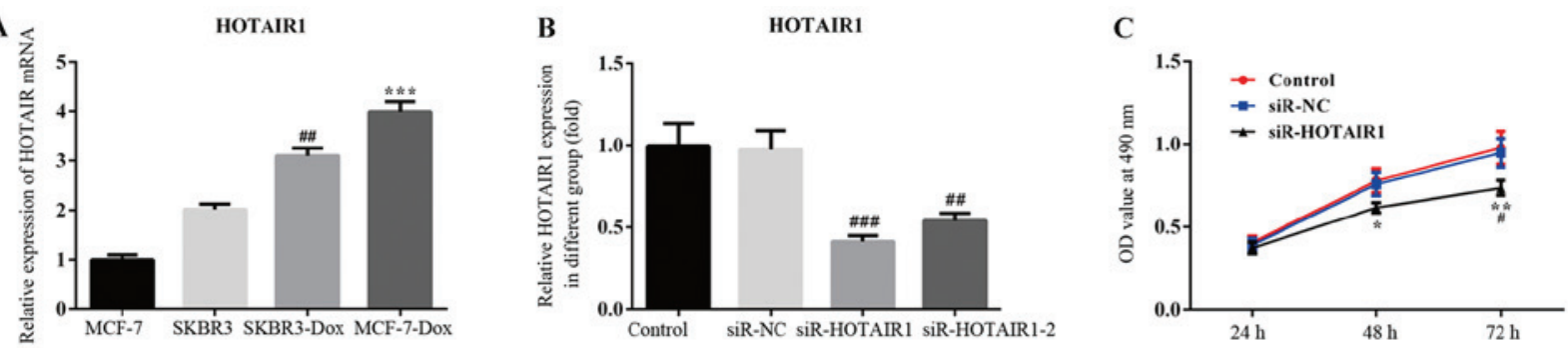

D Control siR-NC
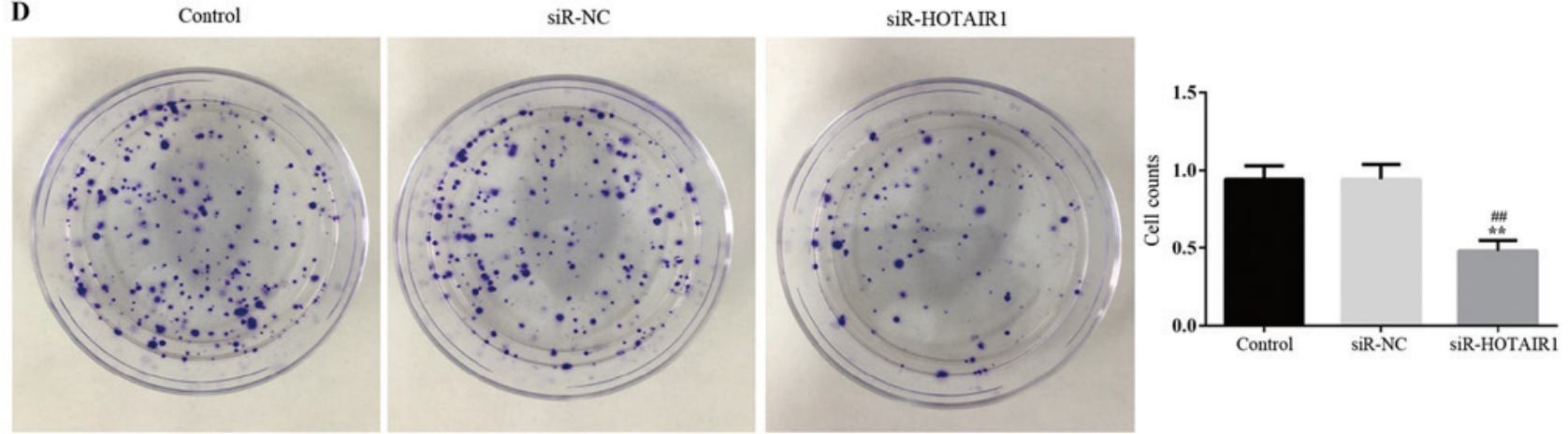

Figure 1. Knockdown of LncRNA-HOTAIR suppressed the proliferation of MCF-7 cells. MCF-7 cells were transfected with lncRNA-HOTAIR siRNA and negative control siRNA for $72 \mathrm{~h}$. (A) Levels of lncRNA-HOTAIR were assessed in the indicated cell lines via RT-qPCR. ${ }^{\# /} \mathrm{P}<0.01 \mathrm{vs}$. the SKBR3 group; ${ }^{* * * *} \mathrm{P}<0.001$ vs. the MCF-7 group. (B) Interference efficiency was also measured via RT-qPCR. ${ }^{\# \#} \mathrm{P}<0.01$ and ${ }^{\# \# \#} \mathrm{P}<0.001$ vs. the NC group. (C) MTT ("P $<0.05$ and ${ }^{* *} \mathrm{P}<0.01$ vs. the $\mathrm{NC}$ group; ${ }^{*} \mathrm{P}<0.05$ vs. the control group) and (D) colony formation assays were performed to assess cell proliferation. The rate of colony formation was quantitatively analyzed. Magnification, $x 100 .{ }^{* *} \mathrm{P}<0.01$ vs. the $\mathrm{NC}$ group; ${ }^{\# \#} \mathrm{P}<0.01 \mathrm{vs}$. the control group. Values are expressed as the mean \pm standard deviation of three independent experiments. LncRNA, long non-coding RNA; HOTAIR, HOX transcript antisense RNA; RT-qPCR, reverse transcription-quantitative polymerase chain reaction; NC, negative control; siRNA, small interfering RNA; Dox, doxorubicin; OD, optical density.

2 (Bcl2; 1:1,000; cat. no. 2872), anti-cleaved caspase3 (1:500; cat. no. 9664), anti-PI3K (1:1,000; cat. no. 4255), anti-AKT (1:500; cat. no. 9272), anti-mTOR (1:500; cat. no. 2972), anti-phospho (p)-mTOR (ser2448; 1;1,000; cat. no. 5536), anti-p-PI3K (tyr458; 1:1000; cat. no. 4228), anti-p-AKT (ser473; 1:500; cat. no. 4060), anti-MRP1 (1:500; cat. no. 14685), anti-multidrug resistance protein 1 (MDR1; 1:500; cat. no. 13978), anti-ATP binding cassette subfamily B member 1 (ABCB1; 1:1,000; cat. no. 12683) and anti-GAPDH (1:2,000; cat. no. 5174). The membrane was then incubated with goat anti-rabbit IgG secondary antibodies (1:3,000; cat. no. ab6721; Abcam, Cambridge, UK) for $1 \mathrm{~h}$ at room temperature. An enhanced chemiluminescence kit (Bio-Rad Laboratories, Inc., Hercules, CA, USA) was used for visualization and the images were analyzed using ImageJ v1.8.0 (National Institutes of Health, Bethesda, MD, USA). The relative quantification of protein expression was analyzed using Image-Pro Plus 6.0 (Media Cybernetics, Inc., Rockville, MD, USA).

Statistical analysis. Statistical analysis was performed using SPSS version 20.0 software (IBM Corp, Armonk, NY, USA). Each experiment was repeated in triplicate and all data are presented as the mean \pm standard deviation. Differences among multiple groups were detected using one-way analysis of variance followed by the Dunnett's post hoc-test. $\mathrm{P}<0.05$ was considered to indicate a statistically significant difference.

\section{Results}

Detection of IncRNA-HOTAIR interference efficiency. The results of RT-qPCR revealed that HOTAIR was upregulated in the drug resistant breast cancer cell line, DOXR-MCF-7, compared with the other cell lines. HOTAIR expression also significantly differed between MCF-7 and MCF-7-DOX cell lines (Fig. 1A). Therefore, MCF-7 and MCF-7-DOX cell lines were used for the subsequent experimentation. The interference efficiency of HOTAIR siRNA was determined via RT-qPCR. The results revealed that the expression of 1ncRNA-HOTAIR1 was significantly reduced in the siR-HOTAIR1 and siR-HOTAIR1-2 groups compared with the negative control (Fig. 1B). The siR-HOTAIR1 plasmid, which exhibited the greatest interference effect, was selected for subsequent experimentation.

Effect of siR-HOTAIR on the proliferation of MCF-7 cells. An MTT assay was performed to assess the proliferation of siR-HOTAIR1-transfected MCF-7 cells using optical density values (Fig. 1C). The results indicated that proliferation was markedly decreased in the siR-HOTAIR1 group compared with the control and NC groups. Subsequently, a colony formation assay was performed to further assess the proliferation rate of MCF-7 cells (Fig. 1D). The results demonstrated that cell colony formation ability was significantly decreased in the siR-HOTAIR1 group compared with the control and NC groups. Furthermore, the results of flow cytometry revealed that the rate of apoptosis was increased in the siR-HOTAIR1 group compared with the control and NC groups (Fig. 2). These results indicate that the knockdown of lncRNA-HOTAIR may suppress the proliferation of MCF-7 cells.

HOTAIR silencing reduces the sensitivity of drug resistance in drug-resistant MCF-7 cells. DOXR-MCF-7 cells were 

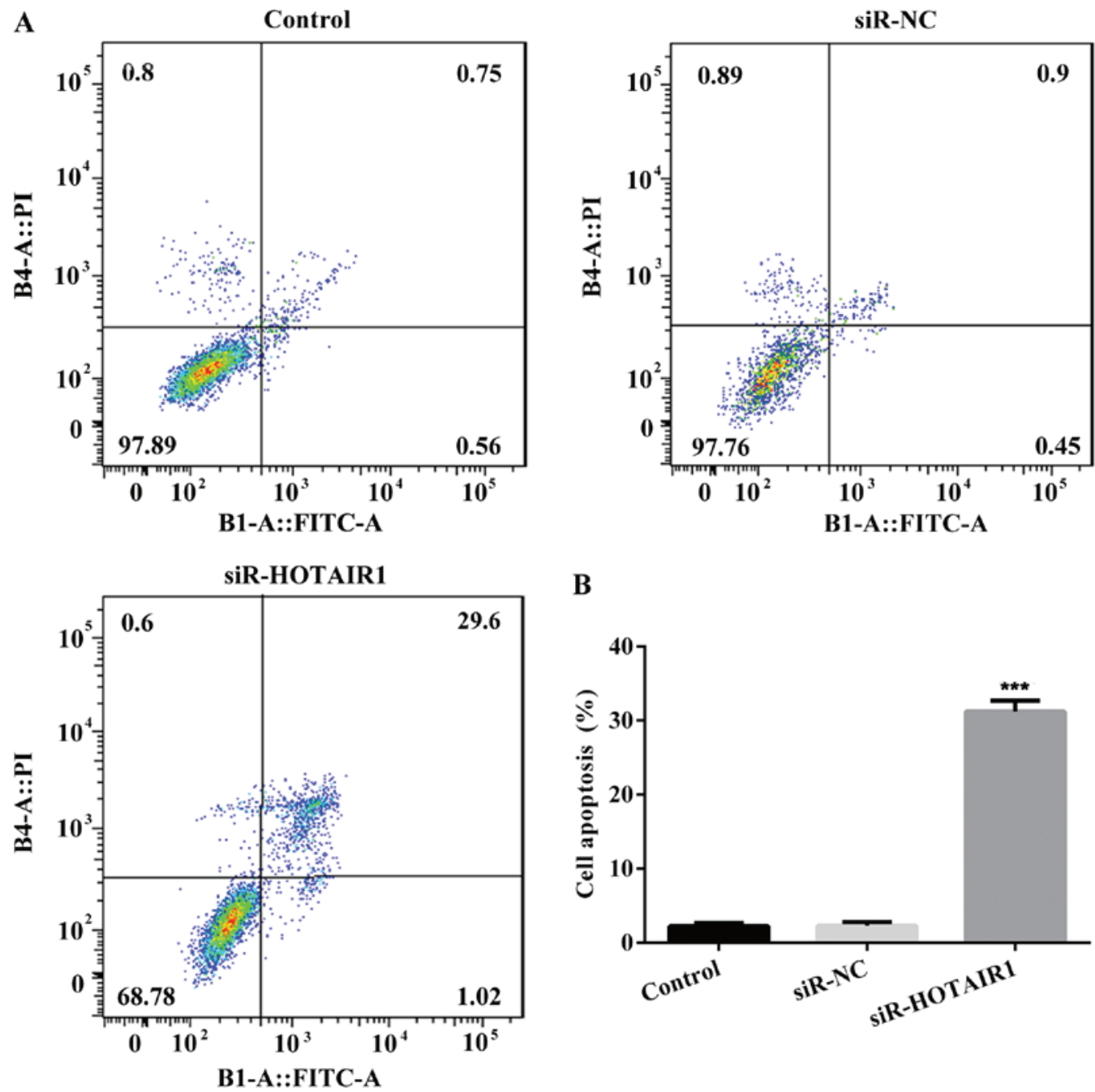

B

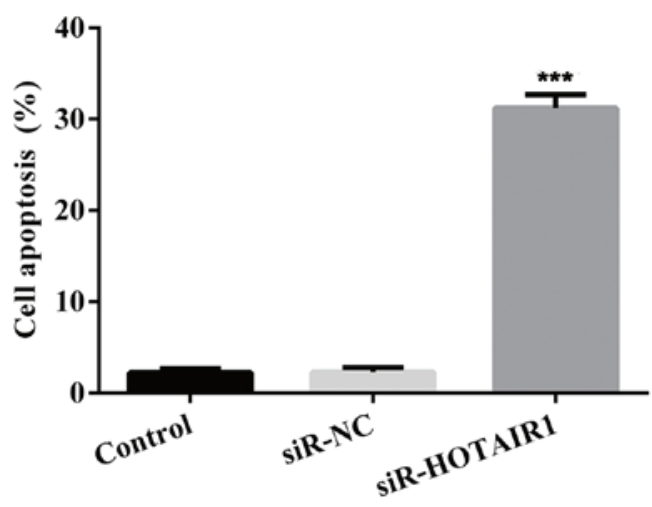

Figure 2. Knockdown of lncRNA-HOTAIR induces the apoptosis of MCF-7 cells. Following staining with Annexin V FITC and PI, apoptotic cells were analyzed using a (A) flow cytometer with (B) subsequent quantification. The Annexin $\mathrm{V}$ assay was performed three times and values are expressed as the mean \pm standard deviation of three independent experiments. ${ }^{* * *} \mathrm{P}<0.001$ vs. the NC group. LncRNA, long non-coding RNA; HOTAIR, HOX transcript antisense RNA; FITC, fluorescein isothiocyanate; PI, propidium iodide; siRNA, small interfering RNA; NC, negative control.

transfected with siR-HOTAIR1 and an MTT assay revealed that proliferation was significantly decreased in the siR-HOTAIR1 group compared with the control and NC groups (Fig. 3A). Additionally, cell colony formation was significantly decreased in the Dox-siR-HOTAIR1 group (Fig. 3B and C). Furthermore, the apoptosis rate of Dox-siR-HOTAIR1 cells was significantly increased compared with the control group (Fig. 4). The results indicate that IncRNA-HOTAIR may serve a key role in the resistance to DOX in MCF-7 cells.

HOTAIR silencing inhibits proliferation and promotes apoptosis by regulating apoptosis associated proteins. The activation of programmed cell death machinery is considered to be associated with the inhibition of cell proliferation. To assess the effect of siR-HOTAIR1 on cell apoptosis, the expression of apoptosis-associated proteins was determined via western blot analysis. Caspase-3, Bcl-2 and Bax have essential roles in cell apoptosis and tumorigenesis. As demonstrated in Fig. 5, the protein levels of caspase-3 and Bax were increased, and the expression of $\mathrm{Bcl}-2$ was markedly decreased in the DOXR-MCF-7 siR-HOTAIR1 group compared with control group. The results indicated that HOTAIR silencing may affect proliferation and promote apoptosis by regulating the expression of apoptosis-associated proteins.

Effect of HOTAIR silencing on drug-resistant proteins. Classical multidrug-resistant proteins, MDR1, MRP1 and $A B C B 1$ were detected to determine the effect of lncRNA-HOTAIR on the drug resistance of DOXR-MCF-7 cells. The protein levels of MDR1, MRP1 and ABCB1 were significantly decreased in DOXR-MCF-7 siR-HOTAIR1 cells compared with the siR-NC DOXR-MCF-7 cells (Fig. 6).

HOTAIR affects the PI3K/AKT/mTOR signaling pathway in drug-resistant MCF-7 cells. The PI3K/AKT/mTOR pathway serves a key role in cell proliferation, cellular metabolism, protein synthesis and in the cell cycle (14). It is considered to be one of the most frequently mutated pathways in cancer (14). To determine the effect of HOTAIR silencing on the PI3K/AKT/mTOR pathway, the phosphorylation of these proteins were analyzed via western blotting. The protein 

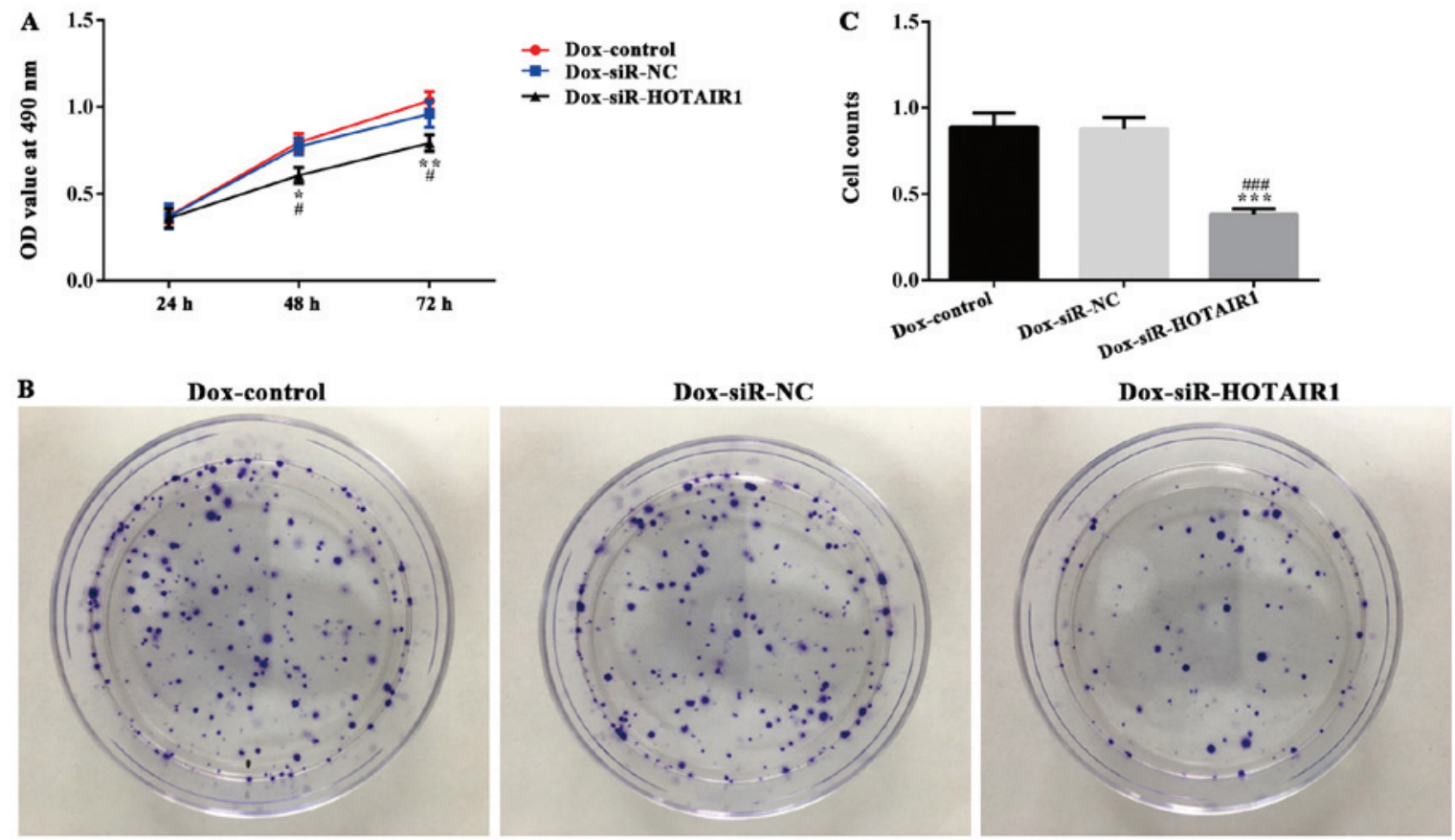

Figure 3. LncRNA-HOTAIR silencing reduces the sensitivity of drug resistance in drug-resistant MCF-7 cells. DOXR-MCF-7 cells were established and transfected with siRNA-HOTAIR1. (A) MTT ("P $<0.05$ and ${ }^{* *} \mathrm{P}<0.01$ vs. the $\mathrm{NC}$ group. ${ }^{*} \mathrm{P}<0.05$ vs. the control group) and (B) colony formation assays with (C) subsequent quantification were performed to assess cell proliferation. ${ }^{* * *} \mathrm{P}<0.001$ vs. the $\mathrm{NC}$ group; ${ }^{\# \# \#} \mathrm{P}<0.001$ vs. the control group. LncRNA, long non-coding RNA; HOTAIR, HOX transcript antisense RNA; DOXR, doxorubicin resistant; siRNA, small interfering RNA; NC, negative control.

A
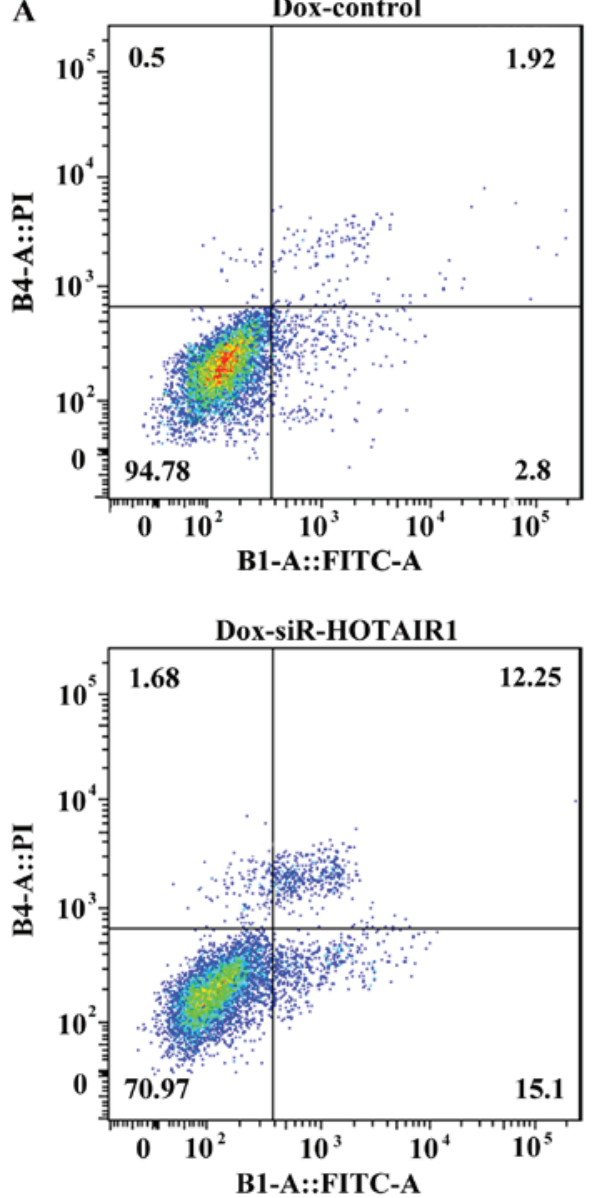

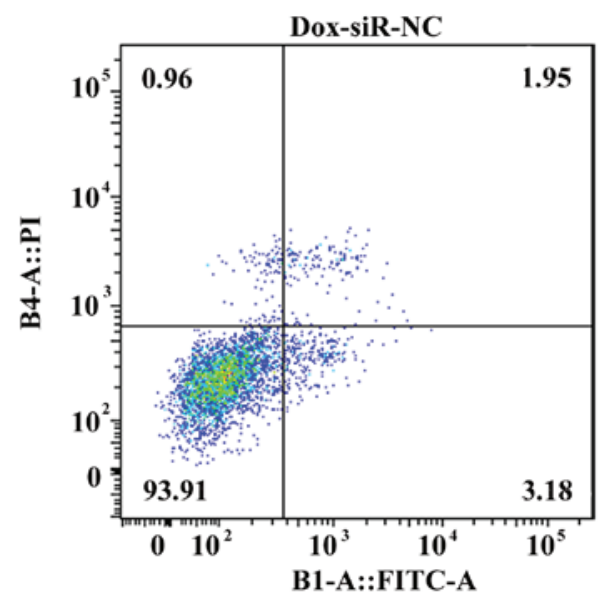

B

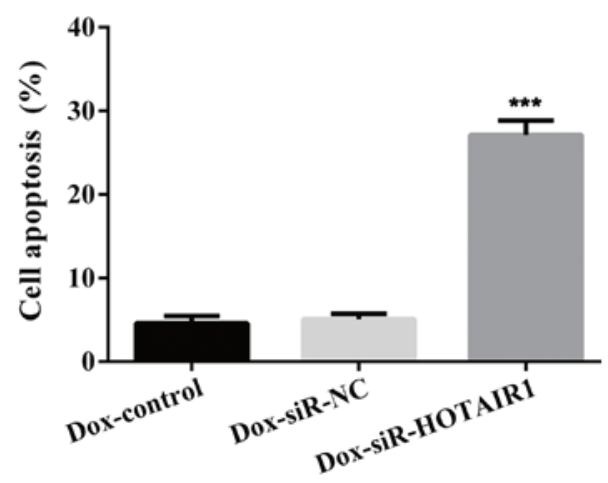

Figure 4. LncRNA-HOTAIR silencing affects the apoptosis of DOXR-MCF-7-cells. After staining with Annexin V FITC and PI, apoptotic cells were analyzed using a (A) flow cytometer with (B) subsequent quantification. The Annexin $\mathrm{V}$ assay was performed three times and values are expressed as the mean \pm standard deviation of three independent experiments. ${ }^{* * *} \mathrm{P}<0.001$ vs. the NC group. LncRNA, long non-coding RNA; HOTAIR, HOX transcript antisense RNA; DOXR, doxorubicin resistant; FITC, fluorescein isothiocyanate; PI, propidium iodide; siRNA, small interfering RNA; NC, negative control. 

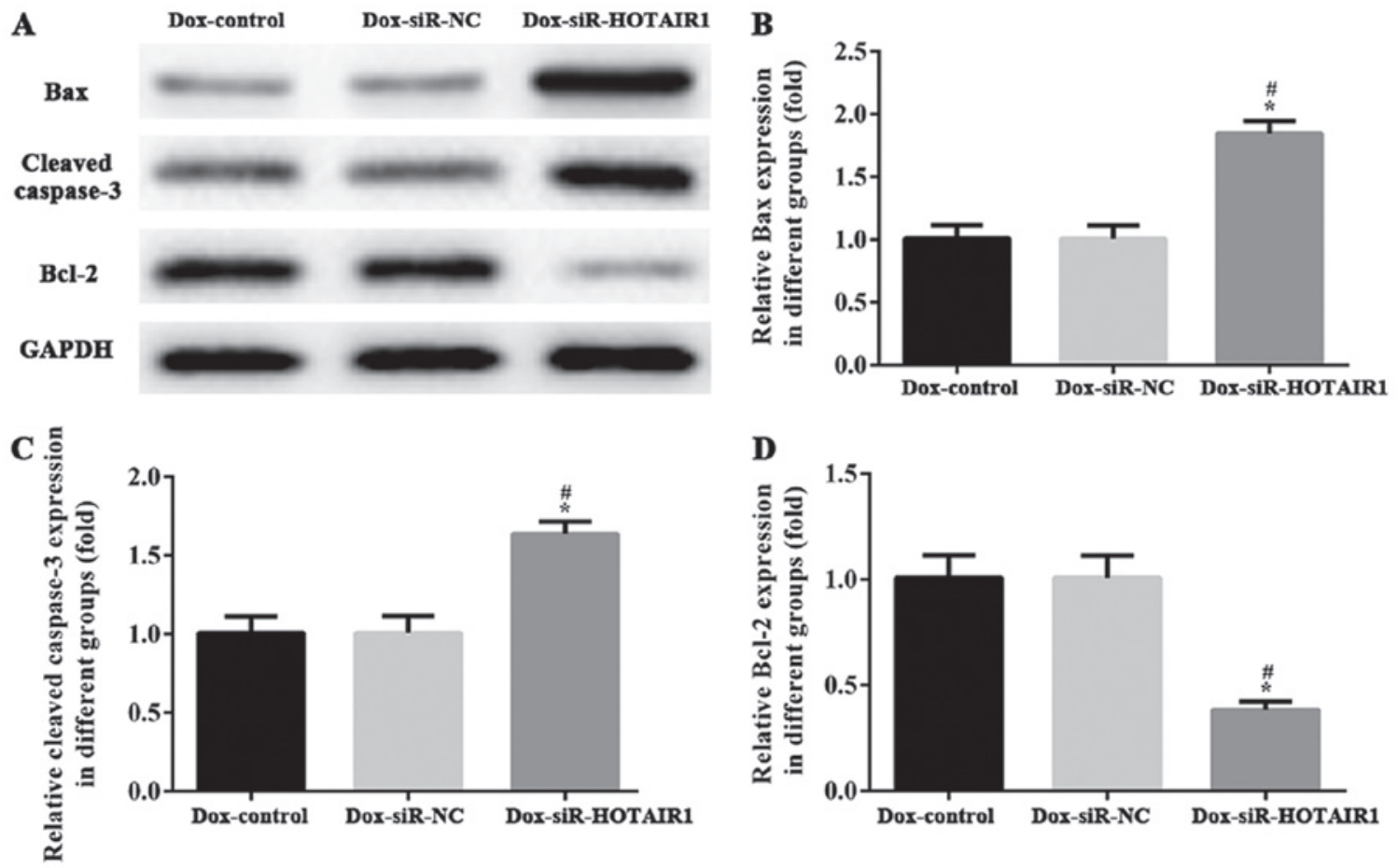

Figure 5. LncRNA-HOTAIR silencing inhibits proliferation and promotes apoptosis by regulating apoptosis associated proteins. (A) The protein expression of (B) Bax, (C) caspase-3 and (D) Bcl-2 and were determined via western blotting. Values are expressed as the mean \pm standard deviation of three independent experiments. " $\mathrm{P}<0.05$ vs. the NC group and ${ }^{*} \mathrm{P}<0.05$ vs. the control group. LncRNA, long non-coding RNA; HOTAIR, HOX transcript antisense RNA; Bax, Bcl-2 associated X; VCl-2, B-cell lymphoma 2; siRNA/siR, small interfering RNA; NC, negative control; Dox, doxorubicin.
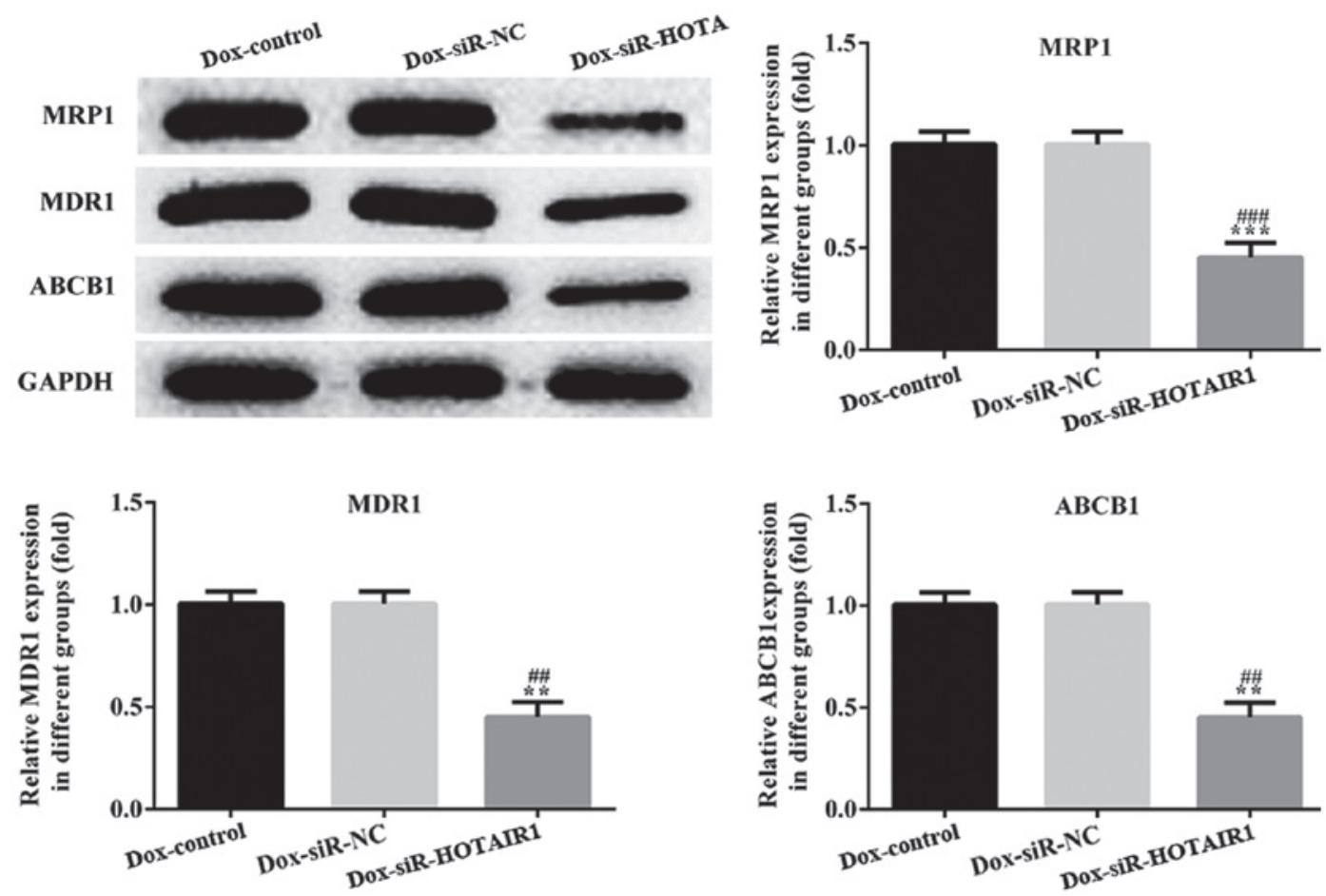

Figure 6. Effect of HOTAIR silencing on drug-resistant proteins. The expression of MDR1, MRP1 and ABCB1 were determined via western blotting. Values are expressed as the mean \pm standard deviation of three independent experiments. ${ }^{* * *} \mathrm{P}<0.001$ and ${ }^{* * *} \mathrm{P}<0.01$ vs. the $\mathrm{NC}$ group. ${ }^{\# \# \#} \mathrm{P}<0.001$ and ${ }^{\# \#} \mathrm{P}<0.01$ vs. the control group. HOTAIR, HOX transcript antisense RNA; MDR1, multidrug resistance protein 1; MRP1, multidrug resistance-associated protein 1; ABCB1, ATP binding cassette subfamily B member 1; NC, negative control; Dox, doxorubicin; siR, small interfering RNA.

expression of PI3K, AKT and mTOR remained unchanged, while the phosphorylation of PI3K, AKT and mTOR was significantly reduced by HOTAIR silencing in DOXR-MCF-7 cells compared with the control and NC groups (Fig. 7). This series of analyses indicate that siR-HOTAIR alters the resistance of MCF-7 cells to DOX. 
$\mathbf{A}$

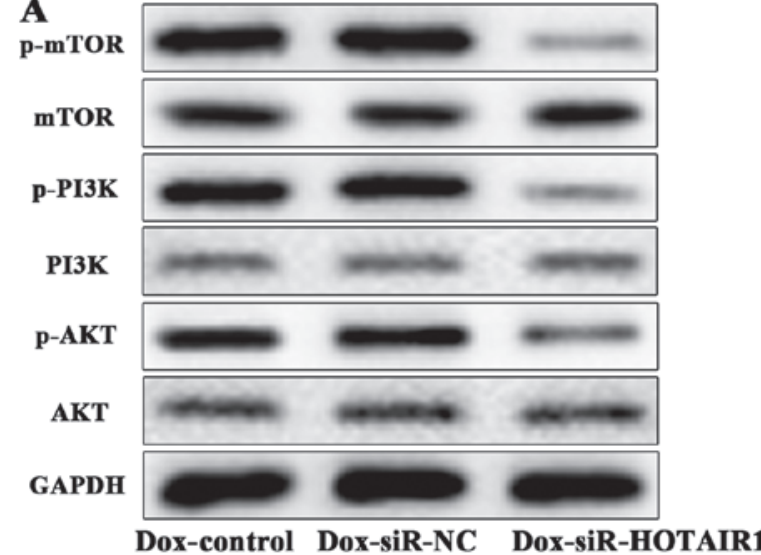

B

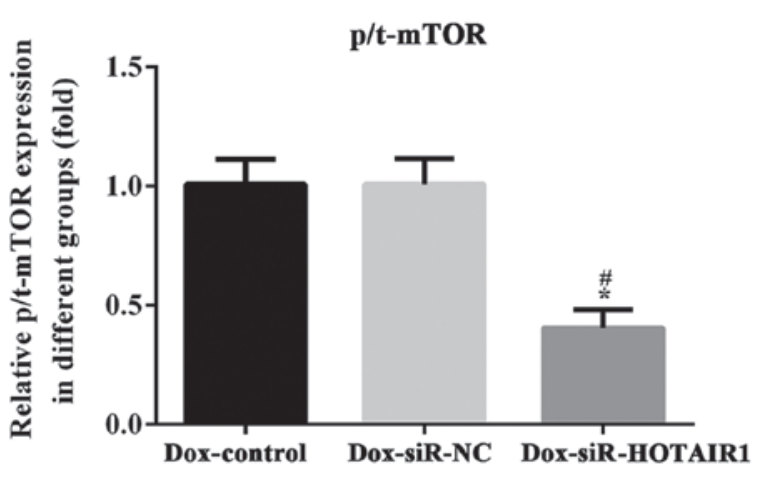

C

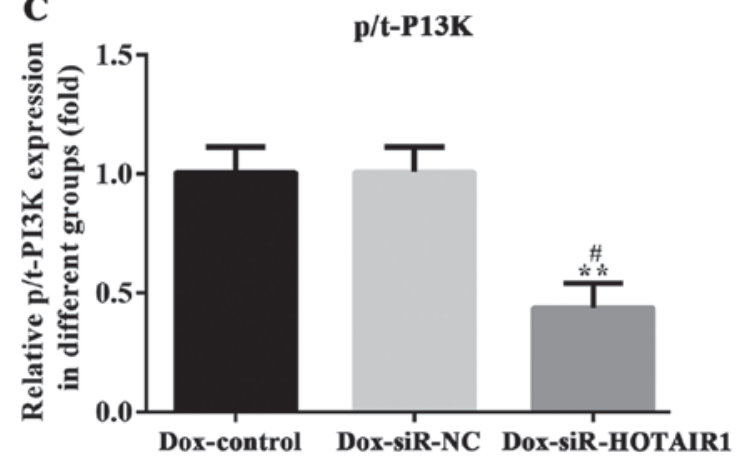

D

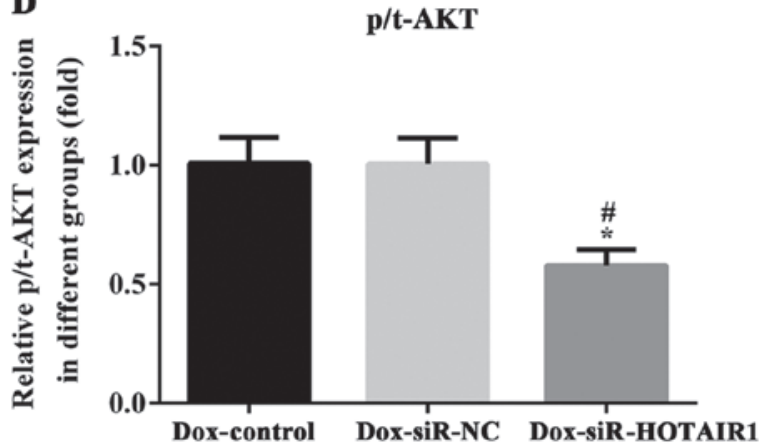

Figure 7. LncRNA-HOTAIR affects the PI3K/AKT/mTOR signaling pathway in DOXR-MCF-7 cells. (A) The protein expression and phosphorylation of (B) mTOR (C) PI3K and (D) AKT was assessed via western blotting. Values are expressed as the mean \pm standard deviation of three independent experiments. ${ }^{*} \mathrm{P}<0.05$ and ${ }^{* *} \mathrm{P}<0.01$ vs. the NC group. ${ }^{*} \mathrm{P}<0.05$ vs. the control group. LncRNA, long non-coding RNA; PI3K, phosphoinositide 3-kinase; AKT, protein kinase B; mTOR, mechanistic target of rapamycin; HOTAIR, HOX transcript antisense RNA; PI3K, siRNA, small interfering RNA; NC, negative control; siR, small interfering RNA.

\section{Discussion}

Breast cancer is the most common cause of cancer-associated mortality among women $(1,2)$ and chemoresistance is a major cause of treatment failure $(19,20)$. Previous research has revealed that drug resistance is associated with DNA repair, aberrant gene expression and epigenetics (21).

LncRNAs have gained increasing attention as they have been reported to be associated with the progression of several types of cancer (22). Previous research has indicated that lncRNAs may be involved in numerous cellular activities and the roles of IncRNAs in various cancer types have been discussed.LncRNA-HOTAIR has been identified as a regulator of trans-silencing (23). A study by Yu et al (24) demonstrated that the knockdown of IncRNA-HOTAIR significantly inhibited MCF-7 cell proliferation and promoted apoptosis (24). The present study revealed that lncRNA-HOTAIR silencing decreased proliferation and increased apoptosis in breast cancer cells, which supports these previous findings.

A previous study has demonstrated that IncRNA-HOTAIR is also involved in regulating the resistance of lung cancer cells to cisplatin (25). Furthermore, previous studies have indicated that the overexpression of HOTAIR promotes the proliferation and enhances doxorubicin resistance in GC and TCC cells; however, HOTAIR silencing exhibits the opposite regulative effects $(26,27)$. In the present study, the expression of multidrug-resistant proteins was significantly decreased by lncRNA-HOTAIR silencing, which also inhibited cell viability and induced apoptosis in DOXR-MCF-7 cells, indicating that the knockdown of IncRNA-HOTAIR decreased DOX resistance in breast cancer cells, which is consistent with previous findings. Furthermore, a previous study demonstrated that IncRNA-HOTAIR silencing suppresses the proliferation and metastasis of osteosarcoma cells by inhibiting the PI3K/AKT/mTOR pathway (28). The results of the present study revealed that the phosphorylation of PI3K, AKT and mTOR were significantly altered in DOXR-MCF-7 cells, which indicated that the knockdown of IncRNA-HOTAIR decreased DOX resistance breast cancer cells by inhibiting the PI3K/AKT/mTOR pathway. Caspase-3, Bcl-2 and Bax serve essential roles in cell apoptosis and tumorigenesis. In the current study, the protein levels of caspase-3, Bcl-2 and Bax were significantly altered by HOTAIR silencing in DOXR-MCF-7 cells, which indicated that the knockdown of IncRNA-HOTAIR promoted apoptosis in DOXR-MCF-7 cells.

In summary, the results of the present study indicated that IncRNA-HOTAIR silencing inhibits cell proliferation and promotes apoptosis in MCF-7 and DOXR-MCF-7 cell lines. Furthermore, the knockdown of IncRNA-HOTAIR reduces DOX resistance in breast cancer cells via the PI3K/AKT/mTOR signaling pathway, indicating that lncRNA-HOTAIR has the potential to become a therapeutic target for the treatment of breast cancer. 


\section{Acknowledgements}

Not applicable.

\section{Funding}

No funding was received.

\section{Availability of data and materials}

The datasets used and analyzed during the current study are available from the corresponding author on reasonable request.

\section{Authors' contributions}

ZL designed the current study. JQ and CZ performed the experiments. JQ and JL analyzed the data. ZL drafted the manuscript and analyzed data. ZL and JQ interpreted data and revised the final manuscript. $\mathrm{ZL}$ wrote the manuscript. All authors read and approved the final manuscript.

\section{Ethics approval and consent to participate}

All experimental protocols were performed in accordance with the principles of the Declaration of Helsinki and were approved by the Clinical Research Ethics Committee of bengbu medical college (Anhui, China).

\section{Patient consent for publication}

Not applicable.

\section{Competing interests}

The authors declare that they have no competing interests.

\section{References}

1. Howell A, Anderson AS, Clarke RB, Duffy SW, Evans DG, Garcia-Closas M, Gescher AJ, Key TJ, Saxton JM and Harvie MN: Risk determination and prevention of breast cancer. Breast Cancer Res 16: 446, 2014.

2. Bargostavan MH, Eslami G, Esfandiari $N$ and Shams Shahemabadi A: MMP9 promoter polymorphism $(-1562 \mathrm{C} / \mathrm{T})$ does not affect the serum levels of soluble MICB and MICA in breast cancer. Iran J Immunol 13: 45-53, 2016.

3. Kakarala M, Rozek L, Cote M, Liyanage S and Brenner DE: Brenner Breast cancer histology and receptor status characterization in Asian Indian and Pakistani women in the U. S. -a SEER analysis. BMC Cancer 10: 191, 2010.

4. Wang H, Yu Y, Jiang Z, Cao WM, Wang Z, Dou J, Zhao Y, Cui Y and Zhang H: Next-generation proteasome inhibitor MLN9708 sensitizes breast cancer cells to doxorubicin-induced apoptosis. Sci Rep 6: 26456, 2016.

5. Palmieri C, Krell J, James CR, Harper-Wynne C, Misra V, Cleator S and Miles D: Rechallenging with anthracyclines and taxanes in metastatic breast cancer. Nat Rev Clin Oncol 7: 561-574, 2010.

6. Wu X, Fu Y, Wang Y, Wan S and Zhang J: Gaining insight into crizotinib resistance mechanisms caused by L2026M and G2032R mutations in ROS1 via molecular dynamics simulations and free-energy calculations. J Mol Model 23: 141, 2017.

7. Guttman M and Rinn JL: Modular regulatory principles of large non-coding RNAs. Nature 482: 339-346, 2012.

8. Chen LL and Zhao JC: Functional analysis of long noncoding RNAs in development and disease. Adv Exp Med Biol 825: 129-158, 2014
9. Liu YR, Tang RX, Huang WT, Ren FH, He RQ, Yang LH, Luo DZ, Dang YW and Chen G: Long noncoding RNAs in hepatocellular carcinoma: Novel insights into their mechanism. World J Hepatol 7: 2781-2791, 2015.

10. Huang T, Alvarez A, Hu B and Cheng SY: Noncoding RNAs in cancer and cancer stem cells. Chin J Cancer 32: 582-593, 2013.

11. Deng G and Sui G: Noncoding RNA in oncogenesis: A new era of identifying key players. Int J Mol Sci 14: 18319-18349, 2013.

12. Gökmen-Polar Y, Vladislav IT, Neelamraju Y, Janga SC and Badve S: Prognostic impact of HOTAIR expression is restricted to ER-negative breast cancers. Sci Rep 5: 8765, 2015.

13. Zhuang Y, Nguyen HT, Burow ME, Zhuo Y, El-Dahr SS, Yao X, Cao S, Flemington EK, Nephew KP, Fang F, et al: Elevated expression of long intergenic non-coding RNA. HOTAIR in a basal-like variant of MCF-7 breast cancer cell. Mol Carcinog 54: 1656-1667, 2015.

14. Yan J, Dang Y, Liu S, Zhang Y and Zhang G: LncRNA HOTAIR promotes cisplatin resistance in gastric cancer by targeting miR-126 to activate the PI3K/AKT/MRP1 genes. Tumour Biol 21: 235-256, 2015.

15. Austreid E, Lonning PE and Eikesdal HP: The emergence of targeted drugs in breast cancer to prevent resistance to endocrine treatment and chemotherapy. Expert Opin Pharmacother 15: 681-700, 2014.

16. Mabuchi S, Kuroda H, Takahashi $\mathrm{R}$ and Sasano T: The $\mathrm{PI} 3 \mathrm{~K} / \mathrm{AKT} / \mathrm{mTOR}$ pathway as a therapeutic target in ovarian cancer. Gynecol Oncol 137: 173-179, 2015.

17. Lee JJ, Loh K and Yap YS: PI3K/Akt/mTOR inhibitors in breast cancer. Cancer Biol Med 12: 342-354, 2015.

18. Livak KJ and Schmittgen TD: Analysis of Relative Gene Expression Data Using Real-Time Quantitative PCR and the 2(-Delta Delta C(T)) method. Methods 25: 402-408, 2001.

19. Amiri-Kordestani L, Basseville A, Kurdziel K, Fojo AT and Bates SE: Targeting MDR in breast and lung cancer: Discriminating its potential importance from the failure of drug resistance reversal studies. Drug Resist Updat 15: 50-61, 2012.

20. Jia H, Truica CI, Wang B, Wang Y, Ren X, Harvey HA, Song J and Yang JM: Immunotherapy for triple-negative breast cancer: Existing challenges and exciting prospects. Drug Resist Updat 32: 1-15, 2017.

21. Jang JE, Eom JI, Jeung HK, Cheong JW, Lee JY, Kim JS and Min YH: Targeting AMPK-ULK1-mediated autophagy for combating BET inhibitor resistance in acute myeloid leukemia stem cells. Autophagy 13: 761-762, 2017.

22. Zhang H, Chen Z, Wang X, Huang Z, He Z and Chen Y: Long non-coding RNA: A new player in cancer. J Hematol Oncol 6: 37, 2013.

23. Woo CJ and Kingston RE: HOTAIR lifts noncoding RNAs to new levels. Cell 129: 1257-1259, 2007.

24. Yu Y, Lv F, Liang D, Yang Q, Zhang B, Lin H, Wang X, Qian G, $\mathrm{Xu}$ J and You W: HOTAIR may regulate proliferation, apoptosis, migration and invasion of MCF-7 cells through regulating the P53/Akt/JNK signaling pathway. Biomed Pharmacother 90: 555-561, 2017.

25. Liu Z, Sun M, Lu K, Liu J, Zhang M, Wu W, De W, Wang Z and Wang R: The long noncoding RNA HOTAIR contributes to cisplatin resistance of human lung adenocarcinoma cells via downregualtion of p21(WAF1/CIP1) expression. PLoS One 8: e77293, 2013.

26. Wang H, Qin R, Guan A, Yao Y, Huang Y, Jia H, Huang W and Gao J: HOTAIR enhanced paclitaxel and doxorubicin resistance in gastric cancer cells partly through inhibiting miR-217 expression. J Cell Biochem 119: 7226-7234, 2018.

27. Shang C, Guo Y, Zhang $\mathrm{H}$ and Xue YX: Long noncoding RNA HOTAIR is a prognostic biomarker and inhibits chemosensitivity to doxorubicin in bladder transitional cell carcinoma. Cancer Chemother Pharmacol 77: 507-513, 2016.

28. Li E, Zhao Z, Ma B and Zhang J: Long noncoding RNA HOTAIR promotes the proliferation and metastasis of osteosarcoma cells through the AKT/mTOR signaling pathway. Exp Ther Med 14: 5321-5328, 2017.

(i) $\Theta$ This work is licensed under a Creative Commons Attribution-NonCommercial-NoDerivatives 4.0 International (CC BY-NC-ND 4.0) License. 